\title{
Serum level of estrogen in Iranian patients with oral lichen planus
}

\author{
Narges Gholizadeh ${ }^{1 \oplus}$, Azadeh Sadeghi ${ }^{2 \oplus}$, Iraj Mirzaii-Dizgah ${ }^{3 \oplus}$, Nafiseh Sheykhbahaei ${ }^{1, *}$
}

\section{Abstract}

Background: Oral lichen planus (OLP) is one of the most common diseases in the oral cavity and has a chronic inflammatory nature. The etiology of this disease remains unclear. OLP is more prevalent in women, but to our knowledge, no study yet evaluated estrogen levels in women with OLP.

Objectives: To determine the serum level of estrogen in female patients with OLP.

Methods: This case-control observational study was conducted in patients who were referred to the Department of Oral and Maxillofacial Medicine at the Tehran University of Medical Sciences. After clinical and histopathological confirmation of OLP diagnosis, and according to exclusion and inclusion criteria, 47 women with OLP and 47 healthy women (10 premenopausal and 37 postmenopausal) were included, as case and control groups, respectively. We obtained blood samples $(5 \mathrm{~mL})$ from each participant to measure the serum level of $17 \beta$-estradiol. Data were analyzed by Spearman rank correlation coefficients and a two-way analysis of variance with Bonferroni post hoc tests.

Results: Serum estrogen level in women with OLP was significantly higher than that in healthy women $(P=0.002)$, and it was also significantly higher in premenopausal women than postmenopausal women $(P<0.001)$. The severity of OLP lesions correlated with estrogen level in postmenopausal women $(r=0.650 ; P<0.001)$; in premenopausal women $(r=0.618 ; P=0.008)$, and in all women with $\operatorname{OLP}(r=0.535 ; P<0.001)$.

Conclusion: OLP, like other autoimmune diseases, is affected by the serum level of sex hormones such as estrogen.

Keywords: estrogen; hormones; Iran; lichen planus, oral; menopause

Oral lichen planus is a chronic autoimmune disease and one of the most common oral mucosal diseases that affect the skin and other mucous membranes such as the vagina, esophagus, and larynx. It is believed that some types of this disease, such as erosive and plaque-like types, are more likely to become malignant [1-3]. The pathogenesis of this disease has considered to be from an inflammatory origin to immunological reactions, but a genetic aspect has also been reported [4]. Degeneration of basal layer keratinocytes by autoreactive cytotoxic $\mathrm{CD}^{+} \mathrm{T}$-cells is considered the main mechanism in the pathogenesis of OLP [3].
Epidemiological studies show greater prevalence of OLP among women than men [5]. It is well known that autoimmune diseases are affected by a background of genetic predisposition, viral infections, sex hormone changes, and stressful lifestyles. According to the clinical observations, autoimmune diseases are more prevalent among women, and this indicates a role for sex hormones in the pathogenesis of this disease. It is generally believed that women and girls of all ages show greater inflammatory responses than men and boys. This is a doubleedged sword that facilitates resistance against infections and sepsis, but may lead to confused immune responses against

* Correspondence to: Nafiseh Sheykhbahaei, Department of Oral and Maxillofacial Medicine, School of Dentistry, Tehran University of Medical Science, Tehran 14399-55991, Iran, email: nsheykhbahaei@sina.tums.ac.ir

'Department of Oral and Maxillofacial Medicine, School of Dentistry, Tehran University of Medical Science, Tehran 14399-55991, Iran

${ }^{2}$ Department of Prosthetics, School of Dentistry, Shahid Beheshti University of Medical Science, Tehran 19839-69411, Iran

${ }^{3}$ Department of Physiology, School of Medicine, Aja University of Medical Sciences, Tehran 14117-18541, Iran

¿ Open Access. ( 2021 Gholizadeh et al., published by Sciendo. (Gc) BY-NC-ND This work is licensed under the Creative Commons Attribution NonCommercial-NoDerivatives 4.0 License. 
self-antigens, which results in a greater prevalence of autoimmune diseases in women than men [6]. Generally, it has been observed that the majority of autoimmune diseases occur when the estrogen level in women changes; such as during the menopause and pregnancy. Estrogen, especially $17 \beta$ estradiol, plays important roles in the oral cavity and immune system through its receptors that are expressed on oral epithelial and immune cells [7]. Estrogen is considered to regulate the activity of the immune system. Three critical factors influence the relationship between estrogen and immune system disease, and these are "estrogen timing," "estrogen concentration," and "estrogen targets." All these 3 factors have been previously studied in research on several types of diseases including systemic lupus erythematosus (SLE), multiple sclerosis, and rheumatoid arthritis (RA) [6].

\section{Objective}

Databases including PubMed, Google Scholar, Scopus, Web of Science (SCI), and Embase with search terms of "lichen planus" OR "oral lichen planus" OR "OLP” AND “estrogen" OR "estradiol" AND "female sex hormone" with time limitation (1900-2016) were searched to collect data on related articles. According to our search, we found no study to determine the relationship between sex hormones and OLP. Therefore, we sought to determine the serum estrogen level and its association with OLP lesions among women patients.

\section{Methods}

The protocol of this study was approved by the Medical Ethics committee of Tehran University of Medical Sciences (approval No. IR.TUMS.VCR.REC.1395.623). The study was conducted in compliance with international guidelines for human research protection, including the principles outlined in the contemporary revision of the Declaration of Helsinki of 1964 incorporating the most recent (2013) and earlier amendments, the Belmont Report, and Council for International Organizations of Medical Sciences (CIOMS) International Ethical Guidelines for Biomedical Research Involving Human Subjects. We included consecutive patients referred to the Department of Oral and Maxillofacial Medicine at Tehran University of
Medical Sciences from October 2016 to December 2017 who met inclusion criteria. We obtained documented written informed consent from each participant and included in this casecontrol study, 47 women with OLP meeting inclusion criteria as a case group, and 47 healthy women as a control group. Both groups were divided into premenopausal and postmenopausal subgroups consisting of 10 premenopausal and 37 postmenopausal women in each group. The sample size was determined for each group according to a pilot study with $\left(\mathrm{Z}_{1-\alpha / 2}+\mathrm{Z}_{1-\beta}\right)^{2} \times\left(\mathrm{Sd}_{1}{ }^{2}+\mathrm{Sd}_{2}{ }^{2}\right) /\left(\mu_{1} \mu_{2}\right)^{2}$ and considering $\alpha=0.05$ and $\beta=0.2$. Diagnosis of OLP was confirmed based on the clinical (bilateral presence of reticular or popular lesions) and histopathological (band-like infiltration of lymphocyte and hydropic degeneration of basal layer cells) evidence according to modified World Health Organization criteria. We obtained blood samples $(5 \mathrm{~mL})$ that were sent to a laboratory to measure the level of $17 \beta$-estradiol. For each subject in the case group, we matched a healthy woman of similar age and menopausal condition in the control group (Table 1). We selected the control group from companions of patients and faculty staff. The sampling method was simple and nonrandomized in both case and control groups. To ensure the most stable level of serum estrogen, which clearly changes during the menstrual cycle, all blood samples from premenopausal healthy women and patients with OLP were taken only in the follicular phase, which was usually on the 4-8th day of the menstrual cycle. Samples of venous blood were taken from the antecubital vein and then centrifuged at $2000 \mathrm{rpm}$ for $10 \mathrm{~min}$ to separate the hematocrit from the plasma. The samples were then sent to a laboratory within $2 \mathrm{~h}$, where they were stored immediately at $-20{ }^{\circ} \mathrm{C}$ in test tubes containing $3 \%$ citric acid as an anticoagulant. The serum level of estrogen was measured in $\mathrm{pg} /$ $\mathrm{mL}$ using a DRG Estradiol ELISA Kit (Germany), which is a solid phase enzyme-linked immunosorbent assay (ELISA), based on the principle of competitive binding. The normal range of estrogen is $30-400 \mathrm{pg} / \mathrm{mL}$ in premenopausal women and $0-30 \mathrm{pg} / \mathrm{mL}$ in postmenopausal women. The severity of lesions in the case group was classified as from mild-to-severe using a grading system based on the predominant clinical condition in their mouth with 4 categories, namely (1) reticular/ popular, (2) atrophic, (3) erosive/desquamative gingivitis, and (4) bullous/ulcerative.

Inclusion criteria were a clinical and histopathological diagnosis of OLP, normal growth pattern and sexual

Table 1. Age of participants (mean \pm standard deviation) in years

\begin{tabular}{lccc}
\hline & Before menopause & Post menopause & All \\
\hline Healthy women & $41.6 \pm 5.4(\mathrm{n}=10)$ & $55.9 \pm 4.0(\mathrm{n}=37)$ & $52.9 \pm 7.3(\mathrm{n}=47)$ \\
OLP & $43.1 \pm 3.4(\mathrm{n}=10)$ & $57.4 \pm 4.9(\mathrm{n}=37)$ & $54.3 \pm 7.5(\mathrm{n}=47)$ \\
$P$ & 0.47 & 0.17 & 0.35 \\
\hline
\end{tabular}


characteristics, normal history of menstrual cycle in premenopausal and menopausal woman, premenopausal women, while their last parturition was at least 1 year earlier, and for menopausal woman whose last menstruation was at least 1 year earlier.

Exclusion criteria included women with a lichenoid condition, which was caused by drug or contact reactions, with the presence of any inflammation or ulcer except OLP in the oral cavity, with systemic and endocrine disorders, those who had their uterus and ovaries removed, who used topical or systemic corticosteroids in the previous 3 months, who used hormone replacement therapy or an oral contraceptive pill in the previous 6 months, who had a history of abortion, who undertook heavy endurance sports, or who breastfeed in the previous 6 months.

To reward the cooperation of participants, we also measured their blood cholesterol level for no charge. To determine the association of OLP and menopause and also to compare the association of menopause with OLP and control groups, data were analyzed using IBM SPSS Statistics for Windows (version 22). Data were analyzed by Spearman rank correlation coefficients and a two-way ANOVA with a Bonferroni post hoc test. $P<0.05$ was considered significant. We used the STROBE statement cross-sectional study guidelines [8].

\section{Results}

Among 56 patients with a clinical diagnosis of OLP, after biopsy, 3 patients were excluded because of a diagnosis of a lichenoid reaction, according to the modified WHO criteria. Then we assessed 53 remaining patients for systemic, hormonal, and menstrual disorders. According to the exclusion and inclusion criteria, 47 female patients with OLP were included in our study. For the control group, we evaluated 67 volunteers for oral health. We excluded 6 participants because of oral inflammation or oral lesions. We further excluded 14 women because of systemic, hormonal, or menstrual disorders. Finally, we measured the serum level of estrogen in 47 healthy and 47 patients with OLP (10 premenopausal and 37 postmenopausal each group). Their age median was 44 years (range 38-48 years) for premenopausal women and 60 years (range 50-68 years) for postmenopausal women in the case group. The median age of the premenopausal woman in the control group was 42 years (range 33-49 years) and postmenopausal woman 58 years (49-66 years).

Serum estrogen level in patients with OLP was significantly higher than that for healthy women $(P=0.002)$ and it was also significantly higher in premenopausal women than premenopausal women $(P<0.001)$ (Table 2).

The severity of OLP lesions correlated with the estrogen level (in postmenopausal women $[r=0.650 ; P<0.001]$; in premenopausal women $[r=0.618 ; P=0.008]$, and in all patients $[r=0.535 ; P<0.001])$.

\section{Discussion}

Our findings showed that patients with OLP, either premenopausal or postmenopausal, had significantly higher serum levels of estrogen than healthy women. In the present study, nearly $80 \%$ of lichen planus samples were in postmenopausal women. We observed a higher serum level of estrogen in OLP patients was associated with greater severity of the lesions.

The limitations of this study included finding patients with oral lichen planus and healthy people without systemic and hormonal problems. The potential biases of the present study may be, multifaceted and even contradictory effects of sex hormone in various concentrations on different parts of the immune system and other systems. The lack of men in the study due to the low number of men with oral lichen planus can be a potential bias source. We did not consider some of the factors that may affect sex hormones levels such as stress and depression, nutrition, sleep deprivation, and chemical agents in the environment.

Premenopausal women and men with RA showed that patients had higher estradiol serum levels compared with healthy women $[9,10]$. There were also direct correlations between serum estradiol levels and the level of inflammation [10].

It is believed that the inflammatory pathways are affected in a bimodal way by estrogen-estradiol. At premenopausal (high) levels estrogen plays an inhibitory role for main

Table 2. Serum level of estrogen $(\mathrm{pg} / \mathrm{mL})$ in patients with OLP and healthy women (before and after menopause)

\begin{tabular}{|c|c|c|c|}
\hline & Healthy women & Women with OLP & $P$ \\
\hline Before menopause & $58.9 \pm 17.8(n=10)$ & $119.8 \pm 35.2(n=10)$ & $0.002^{*}$ \\
\hline Post menopause & $9.4 \pm 3.2(n=37)$ & $26.1 \pm 6.3(n=37)$ & $0.001^{*}$ \\
\hline
\end{tabular}

The data are expressed as mean \pm standard error of the mean and analyzed by two-way analysis of variance with a Bonferroni post hoc test.

${ }^{*} P<0.05$ was considered statistically significant.

OLP, oral lichen planus. 
proinflammatory pathways (except for B cells, apoptosis, and neoangiogenesis). A shift in the proinflammatory pathways have been associated with postmenopausal (low) levels of estrogen thought to stimulate cellular immunity. Three types of autoimmune diseases are considered to exist, while B cells have a major role in autoimmune disease, the outbreak of disease is hastened by the stimulatory effect of estradiol on humoral immunity in the initial years of reproduction in diseases such as SLE. Although T and B cells have an effect on the pathogenesis of the autoimmune disease, the onset of disease may be due to humoral immunity, the incidence of autoimmune disease in women may be delayed because of an inhibitory effect of estradiol on cellular immunity in the late reproductive or postmenopausal phases. Because the low level of estradiol has a stimulatory effect on cellular immunity, if $\mathrm{T}$ cells play an important role in the incidence of this disease, the commencement of the disease is likely to be during the postmenopausal phase when there is a reduction in estradiol and progesterone levels [11].

The incidence and severity of SLE are associated with estrogen levels in both human and animal studies. Estrogen elevates the level of production of anti-double-stranded DNA antibody $\operatorname{IgG}$ and IgM via peripheral blood mononuclear cells in patients with SLE [12]. Antiestrogens decreased the incidence of the disease in women who were prone to SLE. By contrast, estradiol was directly correlated with the severity of SLE in animals [7]. Similar effects have been reported in Sjögren syndrome [13]. In contrast to SLE, due to the immunosuppressive effects of high estrogen levels on cellular immunity, estrogen is protective against multiple sclerosis non-B-cells and the effect could be explained by the existence of myelin antigen reactive $\mathrm{CD}^{+} \mathrm{T}$-lymphocytes in central nervous system [14]. Similar results have been shown for patients with RA [15]. In OLP, CD8 ${ }^{+}$T-lymphocytes, through their auto-cytotoxic activity, trigger apoptosis of the basal cell layers of the oral epithelium. Other findings support the effect of humoral immunity on the incidence of OLP $[1,16]$. In autoimmune disease mediated by $\mathrm{T}$ and $\mathrm{B}$ cells, estrogen may stimulate the production of autoantibody about one decade before the disease manifests, then aggravation of cellular immune response may cause disease outbreak in the postmenopausal period [6]. This may also be true for the OLP.

Estradiol at periovulatory or pregnancy levels has an immunosuppressive effect on cellular immunity; however, through some proinflammatory mechanisms, it has a role in the development of autoimmune diseases such as OLP. Estrogen reduces apoptosis of immune cells, especially B and $\mathrm{T}$ lymphocytes [7].

Estrogen produces hyperactivity of humoral immunity by promoting immunoglobulin and autoantibody production, and also by increased presentation of plasma cells and autoantibodies on the surface of B cells [17]. The secretion of vascular endothelial growth factor by various cell types is elevated by the proantigenic effects of estradiol. Endothelial cell attachment, proliferation, migration together with the formation of capillary structures are also enhanced by estradiol [18].

Cutolo et al. [19] suggested that inflammatory cytokines such as interleukin (IL)-1, IL-6, and tumor necrosis factor (TNF)- $\alpha$ could activate the enzyme aromatase in the peripheral tissues. Aromatase can convert androgens to estrogen. Because levels of these cytokines are increased significantly in OLP, activation of aromatase and elevation of serum estrogen levels are expected. These mechanisms may operate via a positive feedback loop and this would explain the high levels of estrogen in patients with OLP.

In the present study, we found that the majority of women with OLP were postmenopausal. The initiation phase of the disease can occur many years before the symptomatic phase.

In the postmenopausal period, estradiol at lower concentrations has a strong immunostimulatory effect on cellular immunity via stimulating proinflammatory pathways and cytokines such as TNF- $\alpha$, interferon- $\gamma$, IL-1, IL-6, monocyte chemoattractant protein-1, nitric oxide synthase, nuclear factor $\kappa \beta$, activation of natural killer cells, production of matrix metalloproteinase, and formation of reactive oxygen species, which increases membrane expression of adhesion molecules and cell adhesion to endothelial cells, and inhibits production of anti-inflammatory cytokines such as transforming growth factor- $\beta$, IL-4, IL-10 [6]. Moreover, calcineurin and protein phosphatase $2 \mathrm{~B}$ activity are increased when estrogen is cultured with affected $\mathrm{T}$ cells, but not normal $\mathrm{T}$ cells [20]. The role of calcineurin in the pathogenesis of OLP is supported by a clinical trial and is thought to act by stimulating the transcription of the gene that encodes IL-2, and subsequent T-cell activation and proliferation [21].

The effects of sex hormones on the immune system are not always similar and they may produce a stimulatory or suppressive effect. Many factors influence the outcome of immune responses from the interactions between sex hormones and different tissues, such as hormonal level, duration of exposure, the age of the person, type of immune cell, the existence of costimulatory signals, and the level of receptor expression. Moreover, the role of the environment factors should not be overlooked [17].

We propose the following hypotheses.

- Along with other etiologic factors in OLP, estrogen plays an important role in the onset of disease by promoting mechanisms such as humoral immunity, angiogenesis, and reduction of apoptosis of immune cells during the premenopausal phase, and increases the outbreak of 
disease by stimulating the cellular immune system after menopause.

- Women with higher levels of estrogen either before or after menopause, experience more severe symptoms of oral lesions because estrogen stimulates the humoral and cellular immune system.

- Although oral lichen planus is commonly known as an autoimmune disease caused by cellular immunity and cytotoxic T lymphocytes, considering the effect of estrogen, especially at high premenopausal levels, on the activation and differentiation of B lymphocytes into plasma cells and the secretion and release of specific antibodies, we propose that the role of humoral immunity in the pathogenesis and onset of oral lichen planus is important.

Further case-control studies and clinical trials are required to explain the association between estrogen levels and OLP.

\section{Conclusion}

OLP, like many other autoimmune diseases, is associated with changes in blood levels of sex hormones such as estrogen.

Author contributions. NG and NS contributed substantially to the conception and design of the study. AS collected the data and all authors contributed substantially to its analysis and interpretation. AS, IM-D, and NS drafted the manuscript, and all authors critically revised it for important intellectual content. All authors approved the final version submitted for publication and agree to be accountable for all aspects of the work in ensuring that questions related to the accuracy or integrity of any part of the work are appropriately investigated and resolved, and take responsibility for statements made in the published article.

Acknowledgments. The authors did not receive any specific grant for this research from any funding agency in the public, commercial, or not-for-profit sectors.

Conflict of interest statement. The authors have each completed and submitted an International Committee of Journal Editors Uniform Disclosure Form for Potential Conflicts of Interest. None of the authors has any potential of actual conflict of interest to disclose in relation to the present article.

Data sharing statement. Statistical summaries of data sets generated in the present study are included in the published article. Further details are available from the corresponding author on reasonable request after deidentification of data from any person whose data were included in the study.

\section{References}

[1] Agha-Hosseini F, Moosavi MS, Sadrzadeh Afshar MS, Sheykhbahaei N. Assessment of the relationship between stress and oral lichen planus: a review of literature. J Islam Dent Assoc Iran. 2016; 28:78-85.

[2] Agha-Hosseini F, Sheykhbahaei N, SadrZadeh-Afshar M-S. Evaluation of potential risk factors that contribute to malignant transformation of oral lichen planus: a literature review. J Contemp Dent Pract. 2016; 17:692-701.

[3] Boorghani M, Gholizadeh N, Zenouz AT, Vatankhah M, Mehdipour M. Oral lichen planus: clinical features, etiology, treatment and management; a review of literature. J Dent Res Dent Clin Dent Prospect. 2010; 4:3-9. doi: 10.5681/joddd.2010.002

[4] Katne T, Muppirala AVS, Devaraju RR, Gantala R. A potent regimen for oral lichen planus: a review. Ann Essence Dentist. 2017; 9:7c-11c. doi: 10.5368/aedj.2017.9.2.3.2

[5] Yarnell E, Abascal K. Herbal treatment for lichen planus. Altern Complement Ther. 2010; 16:217-22.

[6] Straub RH. The complex role of estrogens in inflammation. Endocr Rev. 2007; 28:521-74.

[7] Ahmed SA. The immune system as a potential target for environmental estrogens (endocrine disrupters): a new emerging field. Toxicology. 2000; 150:191-206.

[8] von Elm E, Altman DG, Egger M, Pocock SJ, Gøtzsche PC, Vandenbroucke JP; STROBE initiative. The Strengthening the Reporting of Observational Studies in Epidemiology (STROBE) statement: guidelines for reporting observational studies. Int J Surg. 2014; 12:1495-9.

[9] Seriolo B, Cutolo M, Garnero A, Accardo S. Relationships between serum $17 \beta$-oestradiol and anticardiolipin antibody concentrations in female patients with rheumatoid arthritis. Rheumatology (Oxford). 1999; 38:1159-61.

[10] Tengstrand B, Carlström K, Felländer-Tsai L, Hafström I. Abnormal levels of serum dehydroepiandrosterone, estrone, and estradiol in men with rheumatoid arthritis: high correlation between serum estradiol and current degree of inflammation. J Rheumatol. 2003; 30:2338-43.

[11] Calabrese EJ. Estrogen and related compounds: biphasic dose responses. Crit Rev Toxicol. 2001; 31:503-15.

[12] Kanda N, Tamaki K. Estrogen enhances immunoglobulin production by human PBMCs. J Allergy Clin Immunol. 1999; 103:282-8.

[13] Verheul H, Schot L, Schuurs H. Therapeutic effects of nandrolone decanoate, tibolone, lynestrenol and ethylestrenol on Sjögren's syndrome-like disorder in NZB/W mice. Clin Exp Immunol. 1986; 64:243-8.

[14] MacKenzie-Graham AJ, Rinek GA, Avedisian A, Morales LB, Umeda E, Boulat B, et al. Estrogen treatment prevents gray matter atrophy in experimental autoimmune encephalomyelitis. J Neurosci Res. 2012; 90:1310-23. 


\section{RASIAN

[15] Vandenbroucke JP, Witteman JC, Valkenburg HA, Boersma JW, Cats A, Festen JJ, et al. Noncontraceptive hormones and rheumatoid arthritis in perimenopausal and postmenopausal women. JAMA. 1986; 255:1299-303.

[16] Gholizadeh N, Poorfar HK, Taghavi Zenouz A, Vatandoost $\mathrm{M}$, Mehdipour M. Comparison of serum autoantibodies to desmogleins I, III in patients with oral lichen planus and healthy controls. Iran J Pathol. 2015; 10:136-40.

[17] Ansar Ahmed S, Hissong BD, Verthelyi D, Donner K, Becker K, Karpuzoglu-Sahin E. Gender and risk of autoimmune diseases: possible role of estrogenic compounds. Environ Health Perspect. 1999; 107(Suppl 5):681-6.
[18] Losordo DW, Isner JM. Estrogen and angiogenesis: a review. Arterioscler Thromb Vasc Biol. 2001; 21:6-12.

[19] Cutolo M, Capellino S, Sulli A, Serioli B, Secchi ME, Villaggio B, Straub RH. Estrogens and autoimmune diseases. Ann N Y Acad Sci. 2006; 1089:538-47.

[20] Rider V, Jones SR, Evans M, Abdou NI. Molecular mechanisms involved in the estrogen-dependent regulation of calcineurin in systemic lupus erythematosus T cells. Clin Immunol. 2000; 95:124-34.

[21] Vohra S, Singal A, Sharma SB. Clinical and serological efficacy of topical calcineurin inhibitors in oral lichen planus: a prospective randomized controlled trial. Int J Dermatol. 2016; 55:101-5. 